\title{
Demixing versus ordering in hard-rod mixtures
}

\author{
René van Roij and Bela Mulder \\ FOM-Institute for Atomic and Molecular Physics, Kruislaan 407, 1098 SJ Amsterdam, The Netherlands
}

(Received 18 July 1996)

\begin{abstract}
We present a simple treatment of the depletion-driven demixing transition in isotropic and perfectly aligned binary hard-rod mixtures. The simplicity is mainly due to the fact that we use the second virial approximation to the Helmholtz free energy in combination with a convenient Legendre transform. This combination leads to an exact expression for the depletion-driven demixing spinodal in isotropic mixtures of hard rods with different diameters. We show for rod species with the same length that the demixing spinodal is thermodynamically stable with respect to the isotropic-nematic transition, if the diameter ratio is larger than about 5 . We also show that perfectly aligned rod mixtures show a nematic-nematic demixing spinodal, that may preempt the nematicsmectic or the nematic-columnar bifurcation if the size difference between the particles is sufficiently large. [S1063-651X(96)06312-X]

PACS number(s): 61.30.Cz, 64.70.Md, 64.75.+g
\end{abstract}

\section{INTRODUCTION}

Since the work of Onsager in the 1940s [1] and that of Alder and Wainwright in the 1950s [2] it has been known that the short-range repulsions between atoms and molecules can be responsible for ordering in simple and complex fluids. Onsager's theory explains the orientational ordering upon densification of a fluid of rodlike particles as the consequence of hard-core repulsions alone [1], and Alder and Wainwright's computer simulations show positional order in a hard-sphere system at sufficiently high densities [2]. Thus in both studies the short-range repulsions are modeled as hard-core repulsions, while the attractions are neglected completely. It has since been shown that many more ordering phenomena can be expected on the basis of hard-core repulsions alone. For instance, also partially positionally ordered liquid-crystalline phases (e.g., smectic and columnar phases) appear in the phase diagram of hard anisotropic particles $[3,4]$. Thus despite the fact that hard-core fluids are athermal, with the density (or pressure) the only thermodynamic variable, their phase behavior is rich.

The phase behavior of fluids consisting of hard particles is even richer in the case of mixtures of several species. Apart from the fact that the symmetry of ordering in a mixture can be different from that of a pure system, there is also the possibility of a demixing transition into coexisting phases with different compositions and densities. The fact that the presence of different species influences the ordering of hardcore mixtures can be understood intuitively in terms of packing arguments. At first sight, however, there seems to be no mechanism for demixing transitions in hard-core fluids. The reason is that one would only expect a demixing transition if like particles attract or unlike particles repel each other relatively strongly. Neither of these two conditions seems to be fulfilled in hard-core mixtures, since there are no attractions at all and the repulsions, which are only manifest if particles "touch," do not discriminate between the species. In the past few years it has become clear, however, that there are yet demixing mechanisms in binary hard-core mixtures.

One of the best-studied hard-core mixtures is the binary hard-sphere mixture. Even for this relatively simple example of an off-lattice additive hard-core mixture matters have not been resolved completely. In 1964 Lebowitz and Rowlinson concluded, using the Percus-Yevick closure of the OrnsteinZernike (OZ) equation, that there is no fluid-fluid phase separation for any composition, pressure, or size ratio of the spheres [5]. This was confirmed in 1971 by Mansoori et al., who generalized the Carnahan-Starling expressions for pure hard spheres to hard-sphere mixtures [6], and did not find any evidence for a spinodal instability. These findings probably triggered the intuitively attractive picture that hard-core fluids do not demix at all. It was therefore surprising when Biben and Hansen in 1991 provided evidence, based on the self-consistent Rogers-Young closure of the OZ equations, for a demixing instability of binary hard spheres if the diameter ratio is more extreme than 1:5 [7]. This result is qualitatively confirmed in a theoretical study by Lekkerkerker and Stroobants [8], whose analysis also provides a clear physical picture of the demixing transition. These authors make the connection between binary hard-sphere mixtures and colloidpolymer mixtures, the latter of which are known to possibly exhibit a phase separation due to the depletion effect $[9,10]$. In this picture, the excluded volume overlap of phaseseparated large (colloidal) spheres generates sufficient free volume for the small (polymeric) spheres to overcome the loss of entropy of mixing caused by the phase separation. We note that this picture suggests that the depletion mechanism has a more-than-two-body character, since it takes at least two big spheres with overlapping excluded volumes to generate any extra free volume for one single small sphere. The recent theoretical advances in the description of binary hardsphere mixtures are supported by a computer simulation study by Dijkstra and Frenkel [11], who observed phase separation in binary mixtures of hard cubes of different sizes on a lattice. Moreover, in the past few years several experimental studies of mixtures of colloidal hard spheres have revealed evidence in favor of a demixing transition if the size ratio is sufficiently extreme $[12,13]$. From these experiments it seems, however, that the demixing transition is strongly coupled to the freezing transition, and that the actual coexistence is that between a solid phase of primarily big spheres and a fluid phase with primarily small spheres. These obser- 
vations are theoretically supported by Poon and Warren [14] and Rosenfeld [15]. Nevertheless, given the sensitivity of the theories to the adopted closure of the OZ equations, it seems not completely resolved whether or not there is a (metastable) fluid-fluid spinodal in binary mixtures of hard spheres.

At first sight the difficulties encountered in the study of the stability of hard-sphere mixtures suggest that mixtures of aspherical particles are really impossible to treat as yet, since the corresponding $\mathrm{OZ}$ equations lack the radial symmetry and are therefore even harder to deal with than those in the hard-sphere case. However, Onsager has shown that the second virial approximation to systems of hard rods is exact in the limit of very thin hard rods. Therefore the opposite is the case: theories for hard rods are much simpler than those for hard spheres. Straightforward generalizations of Onsager's theory to include binary mixtures of longer and shorter hard rods of the same diameter have therefore been performed by several authors [16-20]. Apart from a number of interesting phenomena related to the isotropic-nematic $(I-N)$ transition (e.g., strong fractionation, widening of coexistence region, reentrant behavior), the most relevant result for the present context is the nematic-nematic $(N-N)$ phase separation if the length ratio of the rods is more extreme than about 1:3 $[19,20]$. Surprisingly, the mechanism behind this demixing transition does not involve the excluded volume interactions directly. Instead, the demixing is driven by the orientational entropy, which can be optimized by phase separating the short rods from the long ones, since the latter impose too strong an alignment on the former. Thus this phase separation is driven by the gain of orientational entropy for the short rods that more than compensates the loss of entropy of mixing caused by the demixing. Obviously, this mechanism is different from the depletion mechanism.

Still one could argue that the depletion mechanism could also play a role in mixtures of anisotropic hard particles, just as it may do in mixtures of hard spheres. We show in this paper that this can actually be the case, not for nematic mixtures of long and short rods, but instead for isotropic mixtures of thin and thick rods, possibly (but not necessarily) of different lengths. A physical realization of such a system is a mixture of colloidal rods and liquid-crystalline polymers, since colloidal particles are much "thicker" than polymers. The theoretical description benefits from the fact that the second virial theory for long hard rods is exact and simple enough to allow for analytic results in the isotropic phase. Therefore our results, when applied to binary mixtures of thick and thin hard rods, are not subject to the reservations encountered in the theories of hard-sphere mixtures, which are sensitive to the adopted closure of the $\mathrm{OZ}$ equation. We do, however, have to consider the possibility of orientational ordering of the rods, analogous to the freezing transition in the case of hard-sphere mixtures. We show that for sufficiently dissimilar particles, the isotropic-isotropic $(I-I)$ demixing preempts the isotropic-nematic transition. This result is consistent with a recent analysis by Sear and Jackson, who considered the extreme case of an infinitesimally thin (ideal) polymeric rod and a thicker colloidal rod [21].

The formalism that we adopt to study isotropic mixtures of rods can equally well be applied to mixtures of perfectly aligned rods of different sizes. The case of identical diam- eters and different lengths has been analyzed to study phase transitions in the well-ordered nematic phase of rods. ${ }^{1}$ It was concluded from both a computer simulation study [25] and a density functional analysis [26] that the nematic-smectic- $A$ $(N-S)$ transition (which is the thermodynamically stable one in the aligned pure rod system [27]) may be preempted by a nematic-columnar $(\mathrm{N}-\mathrm{C})$ transition, if the length ratio of the rods exceeds approximately 2 . The possibility of a nematicnematic demixing transition seems to have been ignored in both Ref. [25] and Ref. [26]. We show that this is no longer justified if the length ratio is more extreme than about 1:5, since then the $N-N$ demixing (probably strongly coupled to the $N-S$ transition) preempts the $N-C$ transition. This result is consistent with that obtained by Koda and Kimura, who considered the $\mathrm{N}-\mathrm{S}$ (but not the $\mathrm{N}-\mathrm{C}$ ) transition of perfectly aligned binary rod mixtures as a function of the length ratio of the rods in detail [28]. In this paper we also consider binary mixtures of perfectly aligned rods of the same length with different diameters.

This paper is organized as follows. In Sec. II we discuss the thermodynamics of binary mixtures, first generally and then explicitly in the second virial approximation. We show very generally that the usual description of the depletion effect is equivalent to the one that we consider convenient. In Secs. III and IV we apply the second virial theory to isotropic and perfectly aligned binary mixtures of hard rods, respectively, and find which particle shape parameters determine the stability of these mixtures. Section V is devoted to conclusions and a discussion of the main results.

\section{THERMODYNAMICS OF BINARY MIXTURES}

\section{A. General considerations}

We are concerned with the phase behavior of binary mixtures, consisting of $N_{\sigma}$ particles of species $\sigma=1,2$ in a volume $V$ at temperature $T$. The appropriate thermodynamic potential of such a system is the Helmholtz free energy $F\left(N_{1}, N_{2}, V, T\right)$. Since we focus on hard-core fluids, the temperature is merely a scale factor which is irrelevant for the thermodynamics. For notational convenience we therefore do not retain the temperature dependence of $F$, and of any of the other thermodynamic potentials that we introduce, in the sequel. Since the Helmholtz free energy is an extensive quantity, it can be written as

$$
F\left(N_{1}, N_{2}, V\right)=N f(x, v),
$$

where $N=N_{1}+N_{2}$ is the (extensive) total number of particles and $f$ the (intensive) Helmholtz free energy per particle, which is a function of the (intensive) composition variable $x=N_{2} / N$ and the (intensive) volume per particle $v=V / N$. From $F$ or $f$ the pressure $P$ can be obtained as

$$
P=-\left(\frac{\partial F}{\partial V}\right)_{N_{1}, N_{2}}=-\left(\frac{\partial f}{\partial v}\right)_{x},
$$

\footnotetext{
${ }^{1}$ One should realize, though, that the thermodynamics of the perfectly aligned rod system is certainly not identical to the extremely well-ordered limit of a freely rotating rod system [20,22-24].
} 
where the variables that are kept fixed are denoted below the brackets. Obviously, we have $P=P(x, v)$ here.

This paper is particularly focused on the stability of binary mixtures of hard-core particles. For that reason we study the stability conditions that follow from the Helmholtz free energy $F[29]$. These conditions can be expressed in terms of $f(x, v)$ as

$$
\begin{gathered}
\left(\frac{\partial^{2} f}{\partial v^{2}}\right)_{x}>0, \\
\left(\frac{\partial^{2} f}{\partial x^{2}}\right)_{v}^{>0} \\
\left(\frac{\partial^{2} f}{\partial v^{2}}\right)_{x}\left(\frac{\partial^{2} f}{\partial x^{2}}\right)_{v}-\left(\frac{\partial^{2} f}{\partial v \partial x}\right)^{2}>0,
\end{gathered}
$$

where the variations with respect to $x$ and $v$ are supposed to be independent. Strictly speaking, the three conditions of (3) are not independent, but for reasons of convention and later convenience we retain all three of them. The first line of Eq. (3) is the mechanical stability condition, since combination with Eq. (2) reveals its equivalence with $(\partial P / \partial v)_{x}<0$. The second line of Eq. (3) is the incompressible mixing stability condition, where "incompressible" refers to the composition fluctuations at constant volume $v$. The third line of Eq. (3) reflects the stability with respect to combined volume and composition fluctuations. This combined stability condition is the most restrictive of the three, since a quadratic term is subtracted from a term that is positive if the mechanical and incompressible mixing stability conditions still hold. For this reason we focus primarily on the physical meaning of this combined stability condition, and assume in the sequel that the mechanical and incompressible mixing stability are satisfied.

We start with the observation that the combined stability condition can be regarded as the compressible mixing stability condition, where the composition fluctuations take place at constant pressure instead of constant volume. This is most easily understood by performing the Legendre transform from Helmholtz to Gibbs free energy, given by $G=F+P V$, with $G=G\left(N_{1}, N_{2}, P\right)=N g(x, P)$. Here we introduced the (intensive) Gibbs free energy per particle $g(x, P)$. Using Eq. (2) and the relation $g=f+P v$, it is easily verified that

$$
\left(\frac{\partial^{2} g}{\partial x^{2}}\right)_{P}=\left(\frac{\partial^{2} f}{\partial x^{2}}\right)_{v}-\left(\frac{\partial^{2} f}{\partial x \partial v}\right)^{2} /\left(\frac{\partial^{2} f}{\partial v^{2}}\right)_{x} .
$$

As a consequence we see that the combined stability condition of Eq. (3) implies that

$$
\left(\frac{\partial^{2} g}{\partial x^{2}}\right)_{P}>0
$$

thereby justifying the nomenclature of compressible mixing stability condition. If we realize that $\left(\partial^{2} f / \partial x \partial v\right)$ $=-(\partial P / \partial x)_{v}$, we see from Eq. (4) that the compressible mixing stability condition tends to be violated by binary mixtures of species with widely different equations of state. In fact, since Eq. (4) shows that $\left(\partial^{2} g / \partial x^{2}\right)_{P}<\left(\partial^{2} f / \partial x^{2}\right)_{v}$, it reveals that compressible binary mixtures violate the stability criteria in general more easily than comparable (hypothetical) incompressible mixtures. This has also been remarked by Sanchez [30] in the context of polymer solutions. Sanchez describes the demixing of these solutions as the consequence of a mismatch in the equation of state of polymers and solvent, which is reflected by large values of $(\partial P / \partial x)_{v}$. This demixing mechanism seems, at first sight, to be very different from that observed in binary hard-core mixtures, in which demixing is usually ascribed to the depletion effect, which yields an effective attraction between particles of one species due to the presence of the other [8]. The fact that the depletion effect is stronger when the size ratio of the particles is more extreme correlates, however, nicely with the mismatch in the equation of state of the smaller and bigger species. In the remainder of this section we show that there is a direct link between demixing induced by the difference in equation of state of the constituent species and that induced by the depletion effect. In fact, we show that the thermodynamic description of the two mechanisms is equivalent.

A convenient way to show the equivalence of the loss of compressible mixing stability and depletion-induced demixing is to introduce an alternative choice to split intensive and extensive variables, combined with another Legendre transform of the Helmholtz free energy. Instead of using $N$ as the extensive variable to scale $F$, as in Eq. (1), we now use $V$. This leads to

$$
F\left(N_{1}, N_{2}, V\right)=V \phi\left(\rho_{1}, \rho_{2}\right),
$$

with $\phi$ the (intensive) Helmholtz free energy density as a function of the number densities $\rho_{\sigma}=N_{\sigma} / V$ for $\sigma=1,2$. Using the relations $\rho_{1}=(1-x) / v, \rho_{2}=x / v$, and $\phi=f / v$, it follows readily that

$$
\begin{aligned}
& \left(\frac{\partial^{2} \phi}{\partial \rho_{1}^{2}}\right)_{\rho_{2}}\left(\frac{\partial^{2} \phi}{\partial \rho_{2}^{2}}\right)_{\rho_{1}}-\left(\frac{\partial^{2} \phi}{\partial \rho_{1} \partial \rho_{2}}\right)^{2} \\
& =v^{4}\left[\left(\frac{\partial^{2} f}{\partial v^{2}}\right)_{x}\left(\frac{\partial^{2} f}{\partial x^{2}}\right)_{v}-\left(\frac{\partial^{2} f}{\partial v \partial x}\right)^{2}\right] .
\end{aligned}
$$

We proceed by calculating the chemical potential $\mu_{1}$ of species $\sigma=1$, given by

$$
\mu_{1}=\left(\frac{\partial F}{\partial N_{1}}\right)_{N_{2}, V}=\left(\frac{\partial \phi}{\partial \rho_{1}}\right)_{\rho_{2}},
$$

with $\mu_{1}=\mu_{1}\left(\rho_{1}, \rho_{2}\right)$. The Legendre transform we perform now yields the (extensive) thermodynamic potential $\Omega=F-\mu_{1} N_{1}$, which describes species $\sigma=1$ grand canonically and species $\sigma=2$ canonically: $\Omega=\Omega\left(\mu_{1}, N_{2}, V\right)$. We exploit the extensiveness of $\Omega$ and define $\Omega=V \omega\left(\mu_{1}, \rho_{2}\right)$, with $\omega$ the intensive thermodynamic potential that satisfies $\omega=f-\mu_{1} \rho_{1}$. Using this relation together with Eq. (8) gives

$$
\left(\frac{\partial^{2} \omega}{\partial \rho_{2}^{2}}\right)_{\mu_{1}}=\left(\frac{\partial^{2} \phi}{\partial \rho_{2}^{2}}\right)_{\rho_{1}}-\left(\frac{\partial^{2} \phi}{\partial \rho_{1} \partial \rho_{2}}\right)^{2} /\left(\frac{\partial^{2} \phi}{\partial \rho_{1}^{2}}\right)_{\rho_{2}}
$$


revealing in combination with Eqs. (4) and (7) the equivalence

$$
\left(\frac{\partial^{2} g}{\partial x^{2}}\right)_{P}=0 \Leftrightarrow\left(\frac{\partial^{2} \omega}{\partial \rho_{2}^{2}}\right)_{\mu_{1}}=0
$$

We have almost reached our goal now. What remains to be shown to prove the equivalence of a compressible demixing instability and a depletion-induced demixing instability is the relation between the second equality of Eq. (10) and the depletion effect. This relation becomes clear by considering the pressure in the $\left(\mu_{1}, \rho_{2}\right)$ ensemble, which can be written as

$$
P=-\left(\frac{\partial \Omega}{\partial V}\right)_{\mu_{1}, N_{2}}=-\omega+\rho_{2}\left(\frac{\partial \omega}{\partial \rho_{2}}\right)_{\mu_{1}}
$$

so that

$$
\left(\frac{\partial P}{\partial \rho_{2}}\right)_{\mu_{1}}=\rho_{2}\left(\frac{\partial^{2} \omega}{\partial \rho_{2}^{2}}\right)_{\mu_{1}} .
$$

From Eqs. (10) and (12) we can conclude that a compressible demixing instability is equivalent to a "van der Waals"-like loop in $P\left(\mu_{1}, \rho_{2}\right)$, the spinodal of which is given by $\left(\partial P / \partial \rho_{2}\right)_{\mu_{1}}=0$. The presence of this loop is then, in analogy with equations of state of pure systems, interpreted as the consequence of attractions between the particles of species $\sigma=2$. In hard-core mixtures, with no bare attractions between particles, these must be "effective" attractions between particles of species $\sigma=2$ caused by the presence of a bath of species $\sigma=1$, characterized by the chemical potential $\mu_{1}$. This attraction is known as depletion-induced attraction [8]. Instead of a liquid-vapor instability, which is the usual nomenclature in the case of a pure system showing a van der Waals loop, we now interpret the instability as a demixing instability, and the spinodal as the demixing spinodal.

The above analysis thus reveals the equivalence of demixing induced by the depletion effect and by the difference in equation of state of the pure species. We exploit this formal equivalence to describe depletion-induced demixing in binary mixtures treated in the second virial approximation. We show that the usual description of the depletion effect, in terms of $\Omega$, is analytically intractable unless extra approximations are made. In contrast, the description in terms of $G$ and the combined stability criterion can be dealt with without imposing extra assumptions.

\section{B. Second virial approximation}

Here we discuss the implications of the general discussion above for a specific but typical choice of the Helmholtz free energy. Having in mind hard-core fluids, for which the temperature is merely a scale factor of the thermodynamic potentials, we use units in which $k_{B} T=1$, with $k_{B}$ the Boltzmann constant. From now on we use the number density $n=1 / v$ instead of volume per particle $v$ as independent variable. We focus on homogeneous binary fluid mixtures, for which the Helmholtz free energy can be expanded as a virial series in $n$. Retaining only the lowest order correction to the ideal gas contributions yields the so-called second virial approximation

$$
f(x, n)=f_{\text {mix }}(x)+\frac{1}{2} n E(x),
$$

with the ideal gas term $\ln n$, the free energy of mixing

$$
f_{\text {mix }}(x)=x \ln x+(1-x) \ln (1-x),
$$

and the excluded volume terms

$$
E(x)=(1-x)^{2} E_{11}+2 x(1-x) E_{12}+x^{2} E_{22} .
$$

Here $E_{\sigma \sigma^{\prime}}$ is the excluded volume of a $\left(\sigma \sigma^{\prime}\right)$ pair of particles, given by the negative of the orientation averaged spatial integral over the Mayer function of the $\left(\sigma \sigma^{\prime}\right)$ pair, as in Mayer's imperfect gas theory [31]. Note that $E_{\sigma \sigma^{\prime}}$ is temperature independent, because the Mayer function of hardcore potentials takes only the values -1 (overlap) and 0 (no overlap), independent of temperature. The pressure $P$ in the second virial approximation is given by

$$
P=n+\frac{1}{2} n^{2} E(x)
$$

as follows from inserting Eq. (13) into Eq. (2).

Neglecting the quadratic and higher order terms in $n$ in Eq. (13) is a serious approximation in the case of spherical particles at typical liquid densities. Consequently, this approximation does not yield any reliable thermodynamic information on the binary hard-sphere mixture. However, we show that it does give more insight into the general conditions for demixing. Moreover, and more importantly, there are cases where the second virial approximation is expected to be accurate (e.g., perfectly aligned hard rods [27]) or even exact (e.g., infinitely elongated freely rotating rods [1]). In addition, the structure of the free energy of Eq. (13) is similar to that of mean-field approximations of (incompressible) mixtures on a lattice, if we interpret $n$ as the coordination number of the lattice and $E_{\sigma \sigma^{\prime}}$ as the nearest neighbor interaction energy in units of $k_{B} T$. Examples of such lattice theories are the Bragg-Williams approach to dense liquid mixtures [31] and the Flory-Huggins theory of polymer solutions [31]. Note that these lattice fluids are temperature driven in contrast to the density-driven off-lattice hard particle fluids. Thus although the second virial approximation is not justified in all circumstances, there is reason enough to consider its stability conditions in detail.

When we insert the free energy expression of Eq. (13) into the stability conditions of Eq. (3), and substitute $v=1 / n$ throughout, we obtain

$$
\begin{gathered}
1+n E(x)>0, \\
1-n x(1-x) \chi>0, \\
1+n\left[(1-x) E_{11}+x E_{22}\right]-n^{2} x(1-x) \Delta>0,
\end{gathered}
$$

where we defined

$$
\begin{gathered}
\chi=2 E_{12}-E_{11}-E_{22}, \\
\Delta=E_{12}^{2}-E_{11} E_{22} .
\end{gathered}
$$


We conclude immediately that the mechanical stability condition is always fulfilled for any density $n>0$, since the excluded volume term $E(x)>0$. The incompressible mixing stability condition is not fulfilled for all positive densities if $\chi>0$. In that case, namely, at densities $n>[1 / x$ $+1 /(1-x)] / \chi$, the stability is lost. The physical mechanism behind this instability is provided by the unfavorable interactions between unlike particles, represented by the relatively large excluded volume $E_{12}$ of unlike particles as compared to $E_{11}$ and $E_{22}$ of like particles. It is easily checked that $\chi=-\frac{1}{2} d^{2} E(x) / d x^{2}$, so that an incompressible demixing instability corresponds to a concave function $E(x)$. The fact that the incompressible demixing instability is governed by a linear relation in $E_{\sigma \sigma^{\prime}}$ strongly suggests that direct binary excluded volume interactions drive this instability. An ex- ample of a fluid satisfying $\chi>0$ is the mixture of hard-rodlike and platelike particles of the same volume and aspect ratio, as discussed in [32]. In contrast, binary mixtures of hard spheres have fully convex functions $E(x)$ for any diameter ratio, so that $\chi<0$. In fact, the parameter $\chi$ plays the same role in the present second virial theory as the so-called $\chi$ parameter in Flory's theory of polymer solutions on a lattice [31]. In this lattice theory, the (incompressible) demixing is driven by direct unfavorable nearest neighbor interaction energies between unlike species as compared to that between the like species.

A compressible demixing instability is possible if $\Delta>0$, since then the third inequality of Eq. (17) is no longer satisfied if $n>n_{s}(x)$, where we defined the composition dependent spinodal density

$$
n_{s}(x)=\frac{\left[(1-x) E_{11}+x E_{22}\right]+\sqrt{\left[(1-x) E_{11}+x E_{22}\right]^{2}+4 x(1-x) \Delta}}{2 x(1-x) \Delta} .
$$

The corresponding spinodal pressure $P_{s}(x)$ can be obtained by inserting $n=n_{s}(x)$ into the equation of state, Eq. (16). The critical point of the demixing transition is then obtained from $d P_{s}(x) / d x=0$. The fact that a compressible demixing instability can only occur if $\Delta>0$ indicates that unfavorable excluded volume interactions between unlike particles are responsible again, just as in the case of incompressible demixing. However, the nonlinear dependence of $\Delta$ on $E_{\sigma \sigma^{\prime}}$, and the nonlinearity in $n$ of the compressible mixing stability condition suggest a many-body character for the demixing mechanism. This becomes explicit if we perform the Legendre transform to $g=f+P / n$ in the second virial approximation, yielding the inversion of the pressure-density relation of Eq. (16),

$$
n(x, P)=\frac{-1+\sqrt{1+2 P E(x)}}{E(x)}
$$

from which follows that

$$
g(x, P)=\ln n(x, P)+f_{\text {mix }}(x)+\sqrt{1+2 P E(x)} .
$$

Expanding Eq. (21) in powers of $P$ generates terms of all orders in $P$, even though the second virial approximation of $f$ does not contain quadratic and higher order terms in $n$. The nonlinear terms in $P$ effectively represent the many-body character of the depletion effect, which may drive a demixing transition at sufficiently high $P$ even if $E(x)$ is a convex function. In fact, it turns out that the stability with respect to depletion-driven demixing is governed by the convexity and concavity of $\sqrt{E(x)}$, since one easily checks that $\Delta=-E^{3 / 2}(x) d^{2} \sqrt{E(x)} / d x^{2}$. It is therefore possible that an entirely convex $E(x)$ (with $\chi<0$ and thus no incompressible demixing) can show compressible demixing, because $\sqrt{E(x)}$ is not (entirely) convex. This is just a restatement of our earlier observation that compressible fluids have a stronger tendency to demix than incompressible fluids.
Thus we see from Eq. (21) that a crucial ingredient to obtain higher order terms in $P$ is the nonlinear relation between pressure and density. An important technical aspect is the fact that this relation, given in Eq. (16), could be inverted analytically as in Eq. (20), despite its nonlinearity. This inversion is exactly the problem if the more usual description of depletion, in terms of $\omega\left(\mu_{1}, \rho_{2}\right)$, had been used. To obtain an analytic expression for $\omega$, consistent with the second virial approximation of $f$, would particularly require the inversion $\rho_{1}=\rho_{1}\left(\mu_{1}, \rho_{2}\right)$ from

$$
\mu_{1}\left(\rho_{1}, \rho_{2}\right)=\ln \rho_{1}+\rho_{1} E_{11}+\rho_{2} E_{12} .
$$

Obviously this inversion cannot be performed analytically, unless species $\sigma=1$ is an ideal gas with $E_{11}=0$. This extra assumption need not be made if the theory is based on the Gibbs free energy, or on the combined stability condition.

One should realize that the higher order terms in $P$ in Eq. (21) are modified if higher order terms in the virial series of $f$ are taken into account. It turns out that a third virial theory, which can be dealt with analogously since the corresponding cubic relation between $P$ and $n$ can also be inverted analytically, tends to stabilize a compressible binary mixture, at least to order $P^{2}$ in Eq. (21). This is intimately related to the recent determination of the distance dependent depletion force between the bigger spheres in binary hard-sphere mixtures. Biben and Bladon found in computer simulations that this force is oscillatory with distance [33], and thus contains both repulsive and attractive contributions. This is also found theoretically by Mao, Cates, and Lekkerkerker [34], who showed that only attractions are present to lowest order in the density of small spheres, while higher order terms also contain repulsive contributions. These authors also found a similar effect for the depletion force between big hard spheres in a semidilute solution of small hard rods [35].

We conclude this section with the observation that the demixing spinodal, as follows from the second virial ap- 
proximation to the Helmholtz free energy, can be calculated analytically. Since the second virial approximation is exact for thin hard rods, we have an exact theory for the demixing spinodal in binary mixtures of thin hard rods.

\section{ISOTROPIC MIXTURES OF THIN AND THICK LONG RODS}

We apply the second virial expressions for the demixing instability to a binary fluid mixture consisting of hard spherocylinders of length $L_{\sigma}$ and diameter $D_{\sigma}$ in the isotropic phase. This model may be used as a simplified description of an isotropic binary mixture of colloidal rods of different sizes. If $D_{1} \ll D_{2}$, we may also interpret species 1 as a long stiff liquid-crystalline polymer, and species 2 as a (much thicker) colloidal rod. For convenience we define $L_{2} / L_{1}=\ell, D_{2} / D_{1}=d$, and $b=(\pi / 4) L_{1}^{2} D_{1}$. A conventional dimensionless density is defined as $c=n b$. As indicated above, Onsager showed that the second virial approximation of the free energy of a monodisperse isotropic rod system is exact in the limit of infinite length-to-width ratio [1]. We therefore expect that the second virial approximation of the rod mixture is exact if $L_{\sigma} \gg D_{\sigma^{\prime}}$ for all combinations $\sigma, \sigma^{\prime}=1,2$. In this limit, we may neglect the end-cap contributions to the excluded volume interactions, and we have $E_{\sigma \sigma^{\prime}}=(\pi / 4) L_{\sigma} L_{\sigma^{\prime}}\left(D_{\sigma}+D_{\sigma^{\prime}}\right)$ [1]. Using the scaled units we thus have

$$
\begin{gathered}
E_{11}=2 b, \\
E_{12}=(1+d) \ell b, \\
E_{22}=2 d \ell^{2} b .
\end{gathered}
$$

$$
c_{I-N}(x)=b n_{I-N}(x)=\frac{8}{1+\left(d \ell^{2}-1\right) x+\sqrt{x(1-x)(d-1)^{2} \ell^{2}+\left[1+\left(d \ell^{2}-1\right) x\right]^{2}}} .
$$

It is straightforward to insert (23) into the spinodal expressions $n_{s}(x)$ and $P_{s}(x)$, and study the demixing of the isotropic phase as a function of the relative shapes of the particles, characterized by the ratios $\ell$ and $d$. A first guess of the interesting particle shapes is obtained by inserting Eq. (23) into Eq. (18) to yield

$$
\Delta=(d-1)^{2} \ell^{2} b^{2},
$$

which is manifestly positive if $d \neq 1$. We may therefore expect a demixing transition if $D_{1} \neq D_{2}$, i.e., in mixtures of thin and thick rods. Demixing in the isotropic phase of equally thick long rods is predicted to be impossible in the present theory, even if their lengths are extremely different. We note that the conditions for demixing would have differed substantially if the incompressible criterion had been used, since $\chi=2 b(\ell-1)(1-\ell d)$. Hence an incompressible binary rod mixture could only demix if $\ell>1$ and $d<1 / \ell$, corresponding to mixtures of "thicker shorter" and "thinner longer" rods. This is clearly qualitatively different from what followed from the compressible instability criterion.

So far, we have considered the mixture of rods assuming that it is in the isotropic phase $(I)$. It is well known, however, that hard-rod fluids exhibit a transition to an orientationally ordered nematic phase $(N)$ at sufficiently high densities (or pressures) $[1,4,16]$. It is therefore necessary to investigate whether or not the demixing transition in the isotropic phase, if the particle shapes permit one, is preempted by this $I-N$ transition. An analytic expression for an upper bound of the stability of the isotropic phase can be obtained by a straightforward generalization of the bifurcation analysis of the monodisperse rod system as presented by Kayser and Raveché [36]. Denoting the composition dependent isotropic-nematic bifurcation density by $n_{I-N}(x)$, we obtain
Note that $c_{I-N}(x=0)=4$, consistent with the bifurcation analysis of the monodisperse system by Kayser and Raveché [36]. It is known that the densities of the coexisting isotropic and nematic phase for the system with $x=0$ are given by $c=3.290$ and $c=4.191$, respectively, so that the bifurcation analysis gives a good estimate for the location of the actual phase transition [4]. The pressure at the bifurcation, $P_{I-N}(x)$, is obtained by inserting (25) into (16). In fact, the actual isotropic to nematic transition will already take place at pressures below $P_{I-N}$, but analogous to pure rod systems we expect that $P_{I-N}$ is a good estimate for the true transition pressure. Thus we expect a demixing transition in the isotropic phase of binary mixtures of hard rods if the critical point $\left(x_{c}, P_{c}\right)$ of the demixing transition satisfies $P_{c}<P_{I-N}\left(x_{c}\right)$. We study this criterion for several rod shapes.

First we focus on rods of equal length, so that $\ell=1$, and we consider several values of the diameter ratio $d>1$. In Fig. 1 we plot the spinodal pressure $P_{s}(x)$ and the upper bound of the isotropic-nematic transition pressure $P_{I-N}(x)$ for $d=2$,
4,6 , and $\infty$. The demixing transition is preempted by orientational ordering for $d=2$ and $d=4$, but is thermodynamically stable for the larger values of $d$. We see that the critical point of the demixing transition shifts to smaller values of $x$ when $d$ increases. In the limit of $d \rightarrow \infty$, the critical point is given by $\left(x_{c}, P_{c} b d\right)=(1 / 9,27 / 4)$. This must be compared to the bifurcation pressure $\lim _{d \rightarrow \infty} P_{I-N}\left(x_{c}\right) b d=54$, which is a factor of 8 higher than the critical pressure. We therefore conclude that the demixing sets in well before the orientational ordering if $d$ is sufficiently large, at least larger than about $d \approx 5$. The precise crossover diameter ratio can only be determined by a calculation of the binodals of the $I-I$ transition and the $I-N$ transition, which requires some numerical effort.

Another relatively simple and symmetric case is obtained by setting $E_{11}=E_{22}$, so that $d=1 / \ell^{2}$. If we consider $d>1$ (and $\ell<1$ ), then species 1 may characterize a thin very stiff long polymer and species 2 a much shorter and thicker colloidal rod. We now have $\Delta=(d+1 / d-2) b^{2}$, which is posi- 

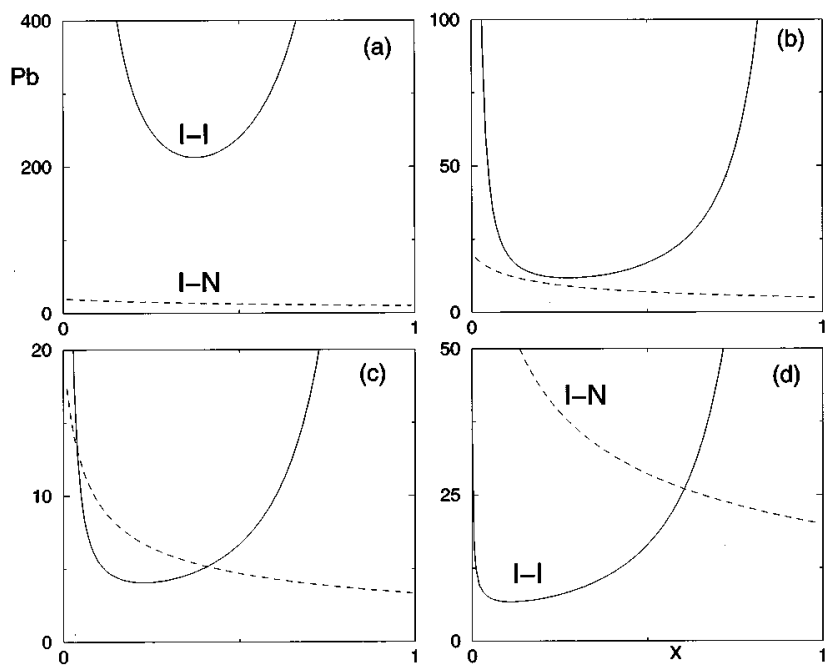

FIG. 1. Phase diagrams of hard rods with the same lengths ( $\ell=1$ ) in the pressure-composition plane for several values of the diameter ratio $d$, (a) $d=2$, (b) $d=4$, (c) $d=6$, (d) $d \rightarrow \infty$. In (d) the vertical axis represents $P b d$, in the other plots $P b$. The continuous curves represent the demixing spinodals in the isotropic phase, the dashed curves the isotropic-nematic bifurcation pressures. For $d=2$ and $d=4$ the demixing transition is thermodynamically metastable with respect to orientational ordering, but at the larger values for $d$ there is a composition regime with a stable $I-I$ phase separation.

tive for any $d \neq 1$. The same trend as found in Fig. 1 is observed now: for small values of $d$ the critical point of the demixing transition is metastable with respect to orientational ordering, while for larger values of $d$ the demixing transition is thermodynamically stable. The value separating the two regimes is given by $d \approx 4.5$. Because of the symmetry, the critical point of the demixing transition is now located at $x=1 / 2$. Recently, Sear and Mulder analyzed this system in more detail, and indeed found $I-I$ demixing to occur [37].

A final illustration of demixing in an isotropic rod mixture is a feeble attempt to describe a mixture of colloidal tobacco mosaic virus (TMV) particles ( $L_{2}=3000 \AA, D_{2}=180 \AA$ ) and a typical stiff 20 beads long liquid-crystalline polymer $\left(L_{1}=20 \times 30=600 \AA, D_{1}=4.5 \AA\right)$. These sizes correspond to $\ell=5$ and $d=40$ in the present notation. In Fig. 2 we show the corresponding spinodal and the isotropic-nematic bifurcation pressure in the composition interval $0<x<0.1$. Clearly, the critical point at $x \approx 0.021$ appears below the nematic ordering bifurcation, hinting at a stable demixing transition in the isotropic phase. Obviously, this result is subject to many reservations. Among them, a condition for the validity of the second virial approximation, $L_{1} \gg D_{2}$, is only marginally satisfied. Moreover, the flexibility and the attractive interactions of the polymers, which are both neglected in the theory, may play significant roles in reality. Still, the basic demixing mechanism described in the present simple theory should be considered in a more elaborate and detailed description of realistic mixtures of rods.

In brief, in this section we have shown that binary mixtures of hard rods with different diameters show an isotropic

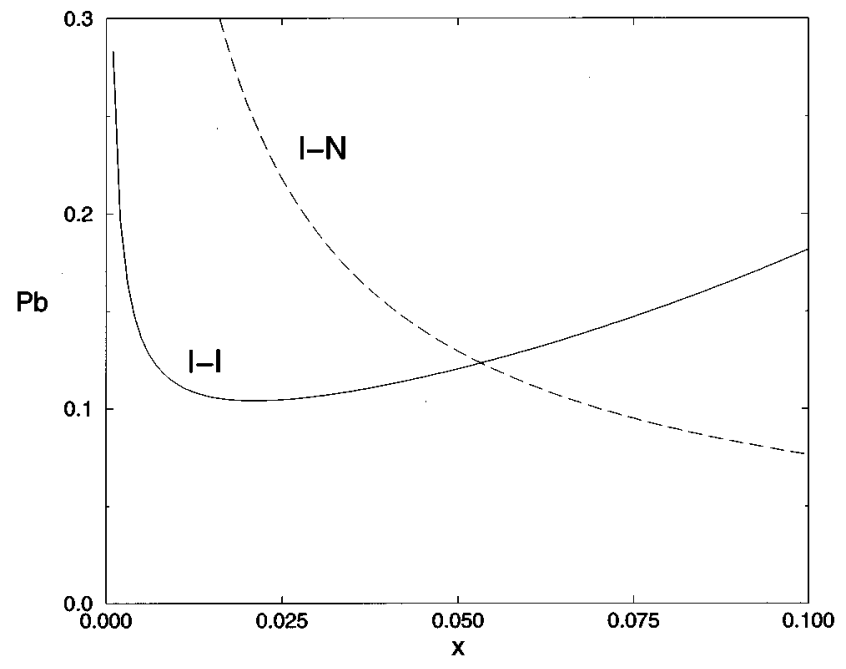

FIG. 2. Phase diagram of large colloidal rods (mole fraction $x$ ) in a stiff liquid-crystalline polymer solution. The shape parameters are $\ell=5$ and $d=40$. The continuous curve represents the demixing spinodal, and the dashed one the isotropic-nematic bifurcation density. The orientational ordering at $x \approx 0.03$ is clearly preempted by demixing into two coexisting isotropic phases of different composition.

demixing spinodal, which preempts the nematic ordering transition if the diameter ratio exceeds a critical value of about 5 .

\section{PERFECTLY ALIGNED ROD MIXTURES}

It is well known that the orientational ordering of rodlike particles in the nematic phase becomes more and more pronounced as the density increases $[1,4,17,19,20]$. Therefore it is tempting to argue that the orientational order becomes saturated at very high densities, and that the well-aligned system can be described assuming perfect alignment of the rods. This assumption, although not entirely justified [20,2224], has led to several interesting predictions concerning the existence of smectic and columnar ordering in dense systems of rods. It was shown by Mulder [27] that a monodisperse aligned rod system exhibits a transition from the nematic phase to the smectic- $A$ phase, characterized by a onedimensional density modulation parallel to the nematic director. An alternative possibility, a transition from the nematic to the two-dimensionally ordered hexagonal columnar phase, was found to be metastable with respect to the nematicsmectic transition. The results of Ref. [27], obtained within the second, third, and fourth virial approximation, are confirmed by more elaborate density functional theories of aligned rods $[38,39]$. There is also agreement with computer simulation studies of this system [40]. Initial doubts as to whether or not orientation fluctuations would destroy the smectic order have been eliminated, since both computer simulation studies [41] and density functional theories $[42,43,24]$ of freely rotating hard rods reveal a stable smectic phase at high densities, although the transition densities differ from those obtained in the aligned system.

Given the qualitatively correct predictions of the aligned monodisperse hard-rod system as a simplification of freely rotating rods at high densities, it should not come as a sur- 
prise that the high density phases of binary hard-rod mixtures have been studied in the same fashion. An interesting result was obtained by Stroobants [25], whose computer simulation study of a binary mixture of longer and shorter aligned hard rods revealed a mechanically stable nematiccolumnar $(N-C)$ transition [and a corresponding metastable nematic-smectic $(N-S)$ transition] if the length ratio of the rods exceeds 1.9 . This was qualitatively confirmed by a density functional analysis by Cui and Chen [26], who adopted the third virial approximation. However, in neither the simulation study of Ref. [25] nor the theoretical study of Ref. [26] was the stability of the homogeneous nematic phase considered. Here we show that a stable nematic phase of a binary mixture of aligned cylinders should not always be taken for granted, as was also concluded in Ref. [28]. A depletion driven nematic-nematic $(N-N)$ demixing transition is shown to possibly preempt the $\mathrm{N}-\mathrm{C}$ and $\mathrm{N}-\mathrm{S}$ transitions if the sizes and shapes of the rods differ substantially.

Using the same notation as above, we can write the second virial approximation to the free energy of a homogeneous binary mixture of $N_{\sigma}$ parallel cylinders of length $L_{\sigma}$ and diameter $D_{\sigma}$ (with $\sigma=1,2$ ) as in Eq. (13), with $E_{\sigma \sigma^{\prime}}=(\pi / 4)\left(L_{\sigma}+L_{\sigma^{\prime}}\right)\left(D_{\sigma}+D_{\sigma^{\prime}}\right)^{2}$. Denoting the volume of the particles of species 1 by $v_{1}=(\pi / 4) L_{1} D_{1}^{2}$, and defining as before the ratios $\ell=L_{2} / L_{1}$ and $d=D_{2} / D_{1}$, we obtain from Eq. (18)

$$
\Delta=\left[(1+d)^{4}(1+\ell)^{2}-64 d^{2} \ell\right] v_{1}^{2} .
$$

Hence $\Delta \geqslant 0$ for any combination of positive values for $\ell$ and $d$, where the equality only holds if $\ell=d=1$. Therefore we expect a demixing transition of the homogeneous aligned hard-rod mixture at sufficiently high densities or pressures. The spinodal pressure $P_{s}(x)$ for a particular set of lengths and diameters of the rods is obtained by inserting the present values for $E_{\sigma \sigma^{\prime}}$ into Eqs. (16) and (19).

The question now is whether or not the $N-N$ demixing transition is preempted by the $\mathrm{N}-\mathrm{S}$ or the $\mathrm{N}-\mathrm{C}$ transition. This can again be sorted out by means of a bifurcation analysis, analogous to the one presented in Ref. [27] for the monodisperse system, and that in Ref. [26] for a binary mixture. Here we only sketch the procedure. The starting point of the analysis is the linearization (about the homogeneous solution) of the stationarity condition of the second virial free energy functional with respect to fluctuations in the oneparticle distributions. Obviously we only consider fluctuations that are consistent with the imposed number density $n$ and composition $x$. If we denote the homogeneous (nematic) distributions by $\rho_{\sigma}=x_{\sigma} n$, with $x_{1}=(1-x)$ and $x_{2}=x$, and the inhomogeneous density fluctuations by $\delta \rho_{\sigma}(\vec{r})$, the stationarity conditions read

$$
\delta \rho_{\sigma}(\vec{r})-\rho_{\sigma} \sum_{\sigma^{\prime}} \int d \vec{r}^{\prime} f_{\sigma \sigma^{\prime}}\left(\vec{r}, \vec{r}^{\prime}\right) \delta \rho_{\sigma^{\prime}}\left(\vec{r}^{\prime}\right)=0
$$

where $f_{\sigma \sigma^{\prime}}$ is the Mayer function of two aligned cylindrical rods of species $\sigma$ and $\sigma^{\prime}$. Note that the linear eigenvalue structure of Eq. (27) is nested, in the sense that $\delta \rho_{\sigma}(\vec{r})$ is an eigenfunction of a $2 \times 2$ problem with respect to $\sigma$, and of a continuous kernel with respect to $\vec{r}$. Using the translational invariance of the Mayer functions, it can be shown that solutions of (27) must be of the form

$$
\delta \rho_{\sigma}(\vec{r})=a_{\sigma} \cos (\vec{q} \cdot \vec{r}),
$$

where we set an arbitrary phase factor to zero, and where the amplitudes $a_{\sigma}$ (possibly negative) and the wave vector $\vec{q}$ $\neq 0$ are unspecified yet. Note that smectic and columnar instabilities are described by $\vec{q} \| \hat{z}$ and $\vec{q} \perp \hat{z}$, respectively, where $\hat{z}$ is the direction of the alignment of the rods. It is easily checked that the trivial solutions $\delta \rho_{\sigma}(\vec{r})=0$ (thus $a_{\sigma}=0$ ) always satisfy Eq. (27). The condition for nontrivial solutions (with $a_{\sigma} \neq 0$ ) is a vanishing determinant of the $2 \times 2$ matrix $\mathbf{B}$, with components

$$
B_{\sigma \sigma^{\prime}}(\vec{q})=\delta_{\sigma \sigma^{\prime}}+n x_{\sigma} M_{\sigma \sigma^{\prime}}(\vec{q}),
$$

where $M_{\sigma \sigma^{\prime}}$ is the negative of the Fourier transform of the Mayer function of aligned cylinders, and $\delta_{\sigma \sigma^{\prime}}$ the Kronecker delta. In terms of the standard and spherical Bessel functions $J_{n}$ and $j_{n}$, respectively, and the definition $T_{n}(y)=J_{n}(y) /$ $\left(\frac{1}{2} y\right)$, we have

$$
M_{\sigma \sigma^{\prime}}(\vec{q})=E_{\sigma \sigma^{\prime}} j_{0}\left[\frac{1}{2}\left(L_{\sigma}+L_{\sigma^{\prime}}\right) q_{z}\right] T_{1}\left[\frac{1}{2}\left(D_{\sigma}+D_{\sigma^{\prime}}\right) q_{y}\right],
$$

where the parallel and perpendicular components of $\vec{q}$ are denoted $q_{z}$ and $q_{y}$, respectively. The smallest density for which the nontrivial solutions exist can now be expressed in terms of the eigenvalues $\Lambda_{i}(\vec{q})$ (with $i=1,2$ ) of the matrix $\mathbf{X M}$, where the diagonal matrix $\mathbf{X}$ has components $X_{\sigma \sigma^{\prime}}=x_{\sigma} \delta_{\sigma \sigma^{\prime}}$. Denoting these minimum $N-S$ and $N-C$ bifurcation densities $n_{N-S}$ and $n_{N-C}$, respectively, we have

$$
\begin{aligned}
& n_{N-S}(x)=\frac{-1}{\min _{q_{z}, i} \Lambda_{i}\left(q_{z}, q_{y}=0\right)}, \\
& n_{N-C}(x)=\frac{-1}{\min _{q_{y}, i} \Lambda_{i}\left(q_{z}=0, q_{y}\right)} .
\end{aligned}
$$

For a given set of particle sizes, the (negative) global minimum of both eigenvalues is easily found numerically. We note that the present bifurcation analysis, which is based on the vanishing determinant of $\mathbf{B}$, is equivalent to the analysis of the divergence of the structure factor matrix $\mathbf{S}$, which is given by $\mathbf{S}(\vec{q})=\mathbf{X B}^{-1}(\vec{q})$ [44]. Using $j_{0}(0)=T_{1}(0)=1$, it can also be seen that the spinodal density $n_{s}(x)$ $=-1 /\left[\min _{i} \Lambda_{i}(\vec{q}=0)\right]$. This can be understood if we realize that $\left(\partial^{2} g / \partial x^{2}\right)_{P}=1 / \lim _{\vec{q} \rightarrow 0} S_{x x}(\vec{q})$, where $S_{x x}$ is a particular combination of the elements of $\mathbf{S}$ [45].

We can now compare the bifurcation densities $n_{N-S}(x)$ and $n_{N-C}(x)$ with each other, and with the spinodal densities $n_{s}(x)$ of the demixing transition. Equivalently, we can compare the corresponding pressures.

The results for aligned cylinders of equal thickness $(d=1)$ and length ratio $\ell=1.8,2,6,10$ are depicted in the pressure-composition plane in Fig. 3. We see that the demixing transition does not interfere with the smectic and columnar ordering for $\ell=1.8,2$, where the critical point of the 

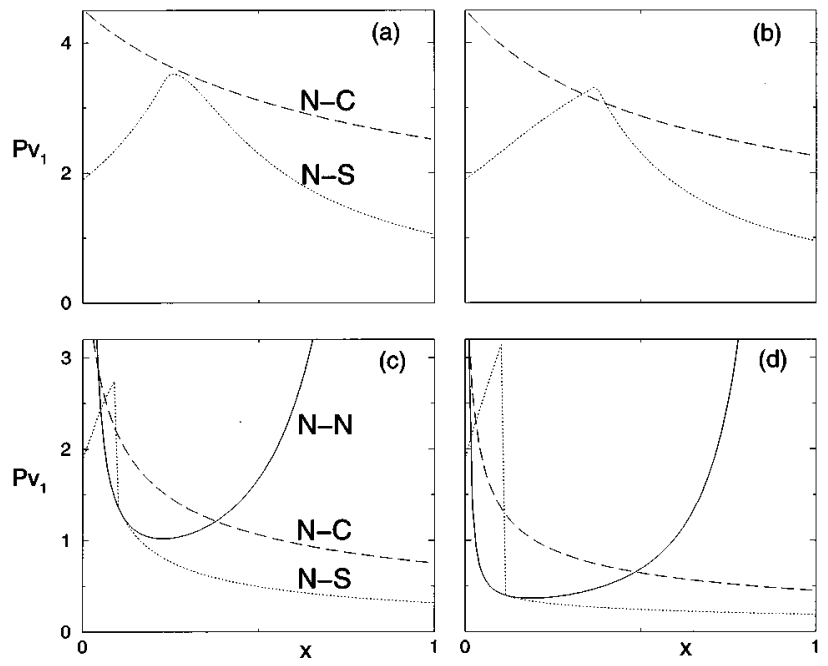

FIG. 3. Phase diagrams of mixtures of equally thick $(d=1)$ aligned short and long hard cylinders, with length ratios (a) $\ell=1.8$, (b) $\ell=2$, (c) $\ell=6$, and (d) $\ell=10$. The continuous curves represent the $N-N$ demixing spinodal, the dotted curves the $N-S$ bifurcation, and the dashed curves the $\mathrm{N}-\mathrm{C}$ bifurcation. The demixing spinodal in (a) and (b) is not plotted, since its critical point is located at $P v_{1} \sim 53$, which is far beyond the present scale. For $\ell=1.8$, there is no stable $N-C$ bifurcation, for $\ell=2$ there is a composition regime where the $N-C$ bifurcation does preempt the $N-S$ bifurcation. For $\ell=6,10$ we see that the $N-C$ bifurcation is metastable with respect to either the $N-N$ demixing or the $N-S$ transition.

spinodal would appear far beyond the scale of the plot at $P v_{1} \sim 53$. For $\ell<1.85$ the $N-S$ transition always preempts the $N-C$ transition, whereas for $\ell>1.85$ a finite regime of $N-C$ transitions is present. However, for the even larger length ratios $\ell=6,10$ the demixing does interfere with the ordering transitions. Actually, we see that the $\mathrm{N}-\mathrm{C}$ transition is preempted by either the $N-N$ demixing or the $N-S$ ordering. The present analysis is not sufficient to determine the precise phase diagram for $\ell>6$, since the $N-N$ and the $N$ $S$ transitions appear at approximately the same pressures. This suggests possible biphasic equilibria between a nematic and a smectic phase, or between two smectic phases, or maybe even a triphasic equilibrium, analogous to the triphasic equilibrium of one isotropic and two nematic phases in freely rotating rod systems $[17,19]$. Whatever the precise phase behavior will be, the possibility of a demixing transition should definitely be taken into account. The maximum of the $N-S$ bifurcation in (a), that develops into a cusp in (b) and further into a discontinuity in (c) and (d) is a consequence of the different character of the global minimum of the eigenvalues $\Lambda_{i}$ as a function of $q_{z}$. For small length ratios (e.g., $\ell=1.85$ ), there is a continuous shift of the associated wavelength (smectic layer thickness) from $1.40 L_{1}$ to $1.40 L_{2}$, the bifurcation layer thickness of the pure systems [27]. For larger length ratios (e.g., $\ell=4$ ), we find several local minima for $\Lambda_{i}$, corresponding to modulations of different wavelength. The global minimum condition then leads to a cusp. For even larger length ratios $(\ell>5)$, we find the minimizing wave vector associated with the smectic modulation of the longer rods to shift to smaller values of $q_{z}$,
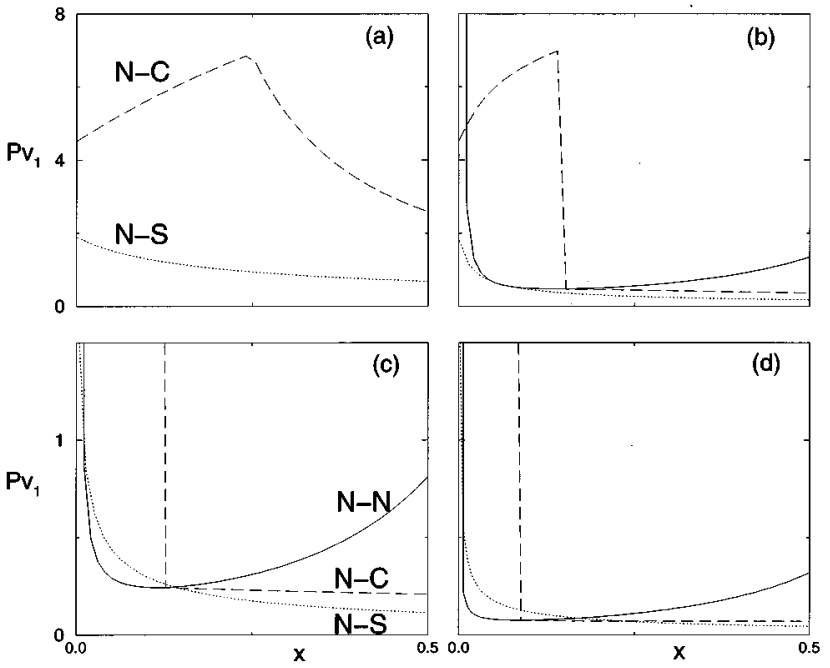

FIG. 4. Pressure-composition phase diagram of mixtures of equally long $(\ell=1)$ aligned thick and thin hard cylinders, with diameter ratios (a) $d=2$, (b) $d=4$, (c) $d=5$, and (d) $d=8$. The nomenclature is as in Fig. 3. We only depict the composition interval $0 \leqslant x \leqslant \frac{1}{2}$, and do not show the $N-N$ spinodal in (a) because it is beyond the scale of the plot. In (a) the $N-S$ bifurcation is stable for all compositions, whereas coupling of all three instabilities seems to occur in (b), (c), and (d). Remarkably, the $\mathrm{N}-\mathrm{C}$ bifurcation preempts the $N-S$ and $N-N$ instability in (d) at $x \approx 0.17$.

eventually reaching $q_{z}=0$. This minimum no longer corresponds to a smectic instability, but rather to the demixing instability, as we argued above. This is a signature of a strong coupling of the $N-N$ and $N-S$ transition, as was also found by Koda and Kimura [28].

We can also consider binary mixtures of equally long aligned rodlike cylinders $(\ell=1)$ with different diameters $(d \neq 1)$. In particular, we present the phase diagram in the pressure-composition plane in Fig. 4, where (a) $d=2$, (b) $d=4$, (c) $d=5$, and (d) $d=8$. Again the continuous curves represent the $N-N$ spinodal, the dotted curves the $N-S$ bifurcation, and the dashed ones the $N-C$ bifurcation. Note that here $0 \leqslant x \leqslant \frac{1}{2}$; for $x>\frac{1}{2}$ the $N-S$ bifurcation is never preempted by the $N-N$ spinodal or the $N-C$ bifurcation. The $N-N$ spinodal is not drawn in (a), since its critical point is beyond the scale of the plot. Clearly, for $d=2$ the $N-S$ bifurcation is the only relevant one, since it preempts the $N$ $C$ and $N-N$ instabilities considerably. For $d=4$, we see that both the $N-N$ spinodal and the $N-C$ bifurcation take place only just above the $N-S$ bifurcation, so that a full calculation may well reveal coexistence between some of these phases. For $d=5$, the $N-N$ spinodal preempts the $N-S$ bifurcation at $x \approx 0.10$, revealing the depletion driven instability of the homogeneous nematic phase. The same holds for $d=8$, with the remarkable difference that the $N-C$ bifurcation preempts the $N-N$ and $N-S$ instability at $x \approx 0.17$. This phenomenon was not observed in Ref. [26], where only diameter ratios between 1.5 and 4 were considered. In fact the same remarks as before can be made about the cusp evolving into a discontinuity, but now for the $N-C$ instead of the $N-S$ bifurcation. The minimizing wave vector of the columnar modulation of the thick particles shifts to smaller $q_{y}$ values and eventually 


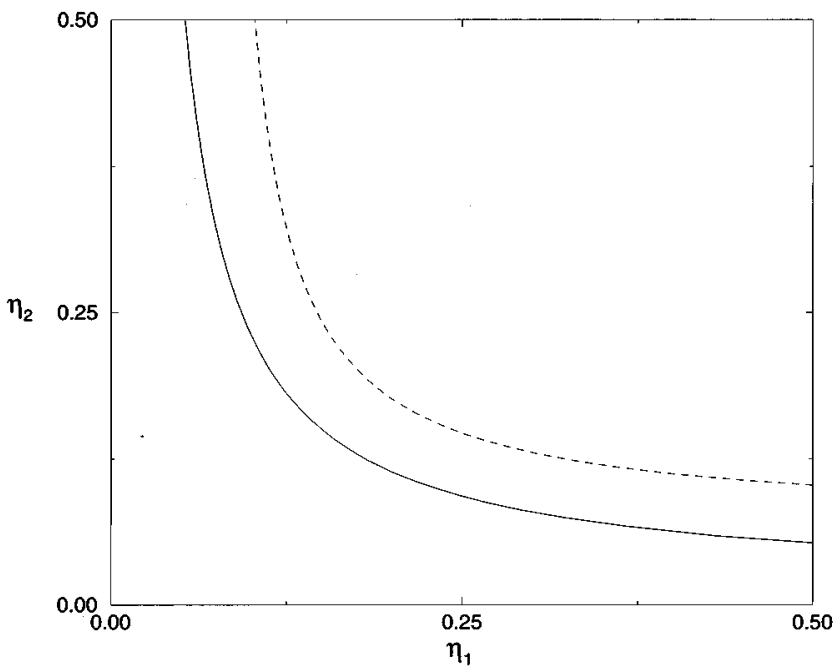

FIG. 5. Comparison of the $N-N$ spinodal in the third (continuous curve) and second (dashed curve) virial approximation of equally thick aligned hard cylinders with length ratio $L_{2} / L_{1}=\ell=9$. Here $\eta_{\sigma}$ denotes the packing fraction of species $\sigma$.

to $q_{y}=0$ if $x$ is reduced. At $q_{y}=0$, the $N-C$ bifurcation condition is identical to that of the $N-N$ demixing spinodal.

We have argued that the second virial approximation gives qualitatively reliable results in the case of perfectly aligned rod mixtures. Still, we expect non-negligible contributions from the higher virial coefficients. In order to illustrate their importance, we have calculated the spinodal of the $N-N$ transition in the third virial approximation of equally thick rods with a length ratio $\ell=9$. A phase diagram of this system, also obtained within the third virial approximation, is presented in Ref. [26]. In the homogeneous phase the dimensionless free energy per particle in the third virial approximation, $f^{(3)}$, reads

$$
f^{(3)}=f^{(2)}+\frac{1}{6} n^{2} E^{(3)}(x),
$$

with $f^{(2)}=f$, the free energy in the second virial approximation as given in Eq. (13), and

$$
\begin{aligned}
E^{(3)}(x)= & \pi^{2}\left(1-\frac{3 \sqrt{3}}{4 \pi}\right) D^{4} \sum_{\sigma, \sigma^{\prime}, \sigma^{\prime \prime}} x_{\sigma^{\prime}} x_{\sigma^{\prime}} x_{\sigma^{\prime \prime}} \\
& \times\left(L_{\sigma} L_{\sigma^{\prime}}+L_{\sigma} L_{\sigma^{\prime \prime}}+L_{\sigma^{\prime}} L_{\sigma^{\prime \prime}}\right) .
\end{aligned}
$$

We used the two-dimensional hard disk diagrams of Ref. [46], and set $D_{1}=D_{2}=D$. Inserting Eq. (32) into the compressible mixing stability condition of Eq. (3) leads to a quartic polynomial in $n$, the relevant root of which can easily be determined to give the spinodal density as a function of composition. For the aligned rod mixture with $\ell=9$ and $d=1$, the resulting third virial spinodal is represented by the continuous curve in Fig. 5, the analogous second virial result by the dashed curve. To facilitate the comparison with Ref. [26], we transformed the variables $(n, x)$ into $\left(\eta_{1}, \eta_{2}\right)$, with the partial packing fractions $\eta_{\sigma}=n x_{\sigma} v_{\sigma}$. Clearly, the inclusion of the third virial term does not alter the demixing phenomenon considerably, except that the spinodal packing fractions are slightly reduced. A comparison with the phase diagrams of Ref. [26] reveals that the $N-S$ and $N-C$ transi- tions can be preempted by $N-N$ demixing. We argue, therefore, that the $\mathrm{N}-\mathrm{N}$ demixing in aligned rod mixtures is not an artifact of the second virial approximation. The results suggest that phase separation in well-ordered nematic mixtures should be considered seriously in the full determination of the phase behavior of these systems.

\section{DISCUSSION}

In this paper we have considered the stability of binary mixtures with respect to demixing into two homogeneous phases, and with respect to possible orientational and positional ordering. Using general thermodynamic relations we argued that a demixing instability is more likely to occur in compressible than in incompressible mixtures. We have also shown that two descriptions of the demixing mechanism - the mismatch in the pure component equations of state and the depletion-induced attraction - just reflect different viewpoints of the same phenomenon. We exploited this equivalence within the second virial theory of the Helmholtz free energy ( $f$ or $\phi)$ of a binary mixture: the Legendre transformation to the Gibbs free energy $g=f+P v$ leads to analytically tractable expressions for the demixing spinodal, while the transformation to the potential $\omega=\phi-\mu_{1} \rho_{1}$, which is usually used to describe depletion, requires the inversion of transcendental relations that cannot be dealt with analytically. The analysis reveals the reason why a second virial approximation to the Helmholtz free energy, which includes only two-body correlations, is still capable of describing a many-body effect as depletion: the resulting Gibbs free energy contains terms of all order in $P$ due to the nonlinear relation between pressure and density.

The demixing spinodal that follows from the second virial theory is calculated analytically for general binary mixtures. We applied the general formalism to describe the isotropic phase of binary mixtures of freely rotating hard rods. Since in the limit of very thin rods the second virial theory is exact, we thus have an exact analytic expression for the spinodal. It turns out that depletion-driven demixing in the isotropic phase is only possible if the diameters of the rods are different, the lengths may but need not be different. In the case of equally long rods, demixing in the isotropic phase preempts nematic ordering if the diameter ratio is more extreme than about 1:5.

We have also applied the second virial approximation to binary mixtures of perfectly aligned hard rods, for which the low density phase is the nematic phase. We found a $N-N$ demixing spinodal for any combination of nonidentical sizes and shapes of the two rod species. If the aligned rods have equal diameters (lengths), this $N-N$ spinodal can preempt the $N-S$ and $N-C$ bifurcation if the length (diameter) ratio is more extreme than about 1:5.

We finally remark that the present theoretical treatment of demixing and ordering is not yet completely conclusive in every detail, since we determined only instability points (the demixing spinodal and the ordering bifurcations), whereas we did not determine the thermodynamic coexistence points. Given the first order nature of the $I-N$ transition, a full calculation of the binodals of the binary mixtures of thick and thin freely rotating hard rods may reveal that the minimum 
diameter ratio required to have a stable $I-I$ binodal is more extreme than the presently quoted value of 1:5. Moreover, such calculations may also reveal a possible three-phasic $I$ $I-N$ equilibrium, analogous to the $I-N-N$ equilibrium in binary mixtures of longer and shorter freely rotating hard rods [19]. In the case of the perfectly aligned mixtures, the coupling between demixing and ordering is probably even stronger, since the $N-N$ spinodal is always very close to the $N$ $S$ and/or $N-C$ bifurcation. Calculations of the binodals may therefore reveal coexistences between nematic, smectic, and/or columnar phases with widely different compositions. Thus although more research is required to map the phase diagrams of binary hard-rod mixtures in full detail, the present results already indicate that spontaneous demixing is a phenomenon to be considered seriously.

\section{ACKNOWLEDGMENTS}

It is a pleasure to thank Henk Lekkerkerker, Daan Frenkel, and Richard Sear for discussions. We are also indepted to Richard Sear for pointing out important references, and for a careful reading of the manuscript. This work is part of the research program of the "Stichting voor Fundamenteel Onderzoek der Materie (FOM)," which is financially supported by the "Nederlandse organisatie voor Wetenschappelijk Onderzoek (NWO).'
[1] L. Onsager, Ann. N.Y. Acad. Sci. 51, 627 (1949).

[2] B.J. Alder and T.E. Wainwright, J. Chem. Phys. 27, 1208 (1957).

[3] M.P. Allen, G.T. Evans, D. Frenkel, and B.M. Mulder, Adv. Chem. Phys. 9, 301 (1992).

[4] G.J. Vroege and H.N.W. Lekkerkerker, Rep. Prog. Phys. 55, 1241 (1992).

[5] J.L. Lebowitz and J.S. Rowlinson, J. Chem. Phys. 41, 133 (1964).

[6] G.A. Mansoori, N.F. Carnahan, K.E. Starling, and T.W. Leland, Jr., J. Chem. Phys. 54, 1523 (1971).

[7] T. Biben and J.P. Hansen, Phys. Rev. Lett. 66, 2215 (1991).

[8] H.N.W. Lekkerkerker and A. Stroobants, Physica A 195, 387 (1993).

[9] S. Asakura and F. Oosawa, J. Chem. Phys. 22, 1255 (1954).

[10] A. Vrij, Pure Appl. Chem. 48, 471 (1976).

[11] M. Dijkstra and D. Frenkel, Phys. Rev. Lett. 72, 298 (1994).

[12] A.D. Dinsmore, A.G. Yodh, and D.J. Pine, Phys. Rev. E 52, 4045 (1995).

[13] A. Imhof and J.K.G. Dhont, Phys. Rev. Lett. 75, 1662 (1995).

[14] W.C.K. Poon and P.B. Warren, Europhys. Lett. 28, 513 (1994).

[15] Y. Rosenfeld, Phys. Rev. Lett. 72, 3831 (1994).

[16] H.N.W. Lekkerkerker, P. Coulon, R. van der Haegen, and R. Deblieck, J. Chem. Phys. 80, 3427 (1984).

[17] T. Odijk and H.N.W. Lekkerkerker, J. Phys. Chem. 89, 2090 (1985).

[18] T.M. Birshtein, B.I. Kolegov, and V.A. Pryamitsyn, Polym. Sci. USSR 30, 316 (1988).

[19] G.J. Vroege and H.N.W. Lekkerkerker, J. Phys. Chem. 97, 3601 (1993).

[20] R. van Roij and B. Mulder, J. Chem. Phys. (to be published).

[21] R.P. Sear and G. Jackson, J. Chem. Phys. 103, 8684 (1995).

[22] D. Frenkel, Mol. Phys. 54, 145 (1984).
[23] A. Poniewierski, Phys. Rev. A 45, 4606 (1992).

[24] A. Poniewierski and T.J. Sluckin, Phys. Rev. A 43, 6837 (1991).

[25] A. Stroobants, Phys. Rev. Lett. 69, 2388 (1992).

[26] S.M. Cui and Z.Y. Chen, Phys. Rev. E 50, 3747 (1994).

[27] B. Mulder, Phys. Rev. A 35, 3095 (1987).

[28] T. Koda and H. Kimura, J. Phys. Soc. Jpn. 63, 984 (1994).

[29] H.B. Callen, Thermodynamics and an Introduction to Thermostatistics, 2nd ed. (John Wiley and Sons, Inc., New York, 1985).

[30] I.C. Sanchez, Macromolecules 24, 908 (1991).

[31] T.L. Hill, An Introduction to Statistical Thermodynamics (Dover Publications Inc., New York, 1986).

[32] R. van Roij and B. Mulder, J. Phys. (France) II 4, 1763 (1994).

[33] T. Biben and P. Bladon (private communication).

[34] Y. Mao, M.E. Cates, and H.N.W. Lekkerkerker, Physica A 222, 10 (1995).

[35] Y. Mao, M.E. Cates, and H.N.W. Lekkerkerker, Phys. Rev. Lett. 75, 4548 (1995).

[36] R.F. Kayser and H.J. Raveché, Phys. Rev. A 17, 2067 (1978).

[37] R. Sear and B. Mulder, J. Chem. Phys. (to be published).

[38] A.M. Somoza and P. Tarazona, Phys. Rev. Lett. 61, 2566 (1988).

[39] R. Holyst and A. Poniewierski, Mol. Phys. 71, 561 (1990).

[40] A. Stroobants, H.N.W. Lekkerkerker, and D. Frenkel, Phys. Rev. Lett. 86, 1452 (1986); Phys. Rev. A 36, 2929 (1987).

[41] J.A.C. Veerman and D. Frenkel, Phys. Rev. A 41, 3237 (1990).

[42] A.M. Somoza and P. Tarazona, Phys. Rev. A 41, 965 (1990).

[43] A. Poniewierski and R. Holyst, Phys. Rev. A 41, 6871 (1990).

[44] J.P. Hansen and I.R. McDonald, Theory of Simple Liquids, 2nd ed. (Academic Press Limited, London, 1990).

[45] A.B. Bathia and D.E. Thornton, Phys. Rev. B 2, 3004 (1970).

[46] P.C. Hemmer, J. Chem. Phys. 42, 1116 (1965). 